\title{
Photoconductivity of activated carbon fibers
}

\author{
K. Kuriyama ${ }^{\text {a) }}$ and M.S. Dresselhaus \\ Center for Materials Science and Engineering, Massachusetts Institute of Technology, \\ Cambridge, Massachusetts 02139
}

(Received 24 August 1990; accepted 1 February 1991)

\begin{abstract}
The conductivity and photoconductivity are measured on a high-surface-area disordered carbon material, i.e., activated carbon fibers, to investigate their electronic properties. This material is a highly disordered carbon derived from a phenolic precursor, having a huge specific surface area of $1000-2000 \mathrm{~m}^{2} / \mathrm{g}$. Our preliminary thermopower measurements show that the dominant carriers are holes at room temperature. The $\mathrm{x}$-ray diffraction pattern reveals that the microstructure is amorphous-like with $L_{c} \simeq 10 \AA$. The intrinsic electrical conductivity, on the order of $20 \mathrm{~S} / \mathrm{cm}$ at room temperature, increases by a factor of several with increasing temperature in the range 30-290 K. In contrast, the photoconductivity in vacuum decreases with increasing temperature. The magnitude of the photoconductive signal was reduced by a factor of ten when the sample was exposed to air. The recombination kinetics changes from a monomolecular process at room temperature to a bimolecular process at low temperatures, indicative of an increase in the photocarrier density at low temperatures. The high density of localized states, which limits the motion of carriers and results in a slow recombination process, is responsible for the observed photoconductivity.
\end{abstract}

\section{INTRODUCTION}

Study of the electronic properties of disordered systems is currently an area of active interest. In the case of carbon materials, interesting phenomena that are not observed in graphitic systems have been reported in some disordered carbons, such as Saran carbon rods (in terms of their conductivity ${ }^{1}$ ), active carbon rods (in terms of their resistivity ${ }^{2,3}$ ), evaporated carbon films (in terms of their resistivity, ${ }^{4,5}$ photoconductivity, ${ }^{6}$ optical absorption edge, ${ }^{7}$ adsorption, ${ }^{8,9}$ and $\mathrm{ESR}^{10}$ ), anthracite carbon powders (in terms of their electrical proper$\operatorname{ties}^{11}$ ), glassy carbons, ${ }^{12-14}$ and others. ${ }^{15-20}$ A series of systematic investigations on the electronic properties of various types of carbon materials have been carried out by Mrozowski. ${ }^{21-27}$

Activated carbon fibers (ACFs) are expected to show electronic behavior characteristic of disordered systems, since ACFs are a highly disordered carbon material with high specific surface areas. As shown in a B.E.T. measurement of other activated carbon materials, ${ }^{1}$ every second atom can be a surface atom that has a dangling bond. Thus activated carbon is regarded as a good material for research on strongly disordered systems. However, few electronic properties of ACFs have been reported so far, though their adsorption properties have been extensively investigated. ${ }^{28-41}$ The adsorption rate of the ACFs is $100-1000$ times faster than granular-

\footnotetext{
${ }^{a)}$ Permanent address: Advanced Carbon Research Laboratory, Sumitomo Metal Industries Ltd., Sunayama 16, Hasaki, Kashima, Ibaraki-ken, Japan.
}

type activated carbons and the adsorption capacity is ten times greater than that for the granular type. ${ }^{33}$ An electric double layer capacitor ${ }^{34}$ is an example of an application taking advantage of the huge specific surface area of ACFs.

The fibers of the present study are derived from phenol, one of four precursors used to prepare ACFs: PAN, ${ }^{28,29}$ cellulose,${ }^{30}$ pitch, ${ }^{31}$ or phenol. ${ }^{32,33}$ To prepare the ACFs, these precursors are activated at temperatures $1100-1400 \mathrm{~K}$ in $\mathrm{O}_{2}, \mathrm{H}_{2} \mathrm{O}, \mathrm{CO}_{2}$, or other oxidizing atmospheres. ${ }^{34-38}$ In contrast with usual heat treatments, the consequence of this heat treatment is to form more disorder in the material. The specific surface area is controlled by changing the temperature and the time for the activation process. The maximum specific surface area is about $2800 \mathrm{~m}^{2} / \mathrm{g}$ in commercial activated carbon fibers. ${ }^{33}$ Among these ACFs, the phenol-derived fibers studied in this paper have a relatively high strength, a large specific surface area, and require a simple process for activation. ${ }^{33}$ They have a diameter of around $10 \mu \mathrm{m}$, a conductivity of the order $20 \mathrm{~S} / \mathrm{cm}$ at room temperature, and a specific surface area in the range $1000-2000 \mathrm{~m}^{2} / \mathrm{g}$.

For ACFs, photoconductive measurements are particularly useful as a characterization technique for the following reasons. Measurement of the Hall effect, which is usually a useful technique to determine the type of carriers and their density, is not feasible because of the very small diameter of the ACFs. Even if the measurement were possible, good data would not be expected because the mobility in such a disordered 
material should be very small. In fact, Steinbeck et al. measured the photocurrent for pitch-based carbon fibers ${ }^{42}$ (more conductive than our ACFs by two orders of magnitude) and found that the magnitude of the photocurrent decreases with increasing heat treatment temperature and increasing $L_{a}$.

In this paper, we first report the results of the conductivity and the photoconductivity measurements of the activated carbon fibers. Then the conduction mechanism is discussed based on their temperaturedependences. The recombination kinetics for the photoconductivity are also examined. In the final section, we quantitatively fit the conductivity data to functional forms appropriate for considering the conduction mechanisms.

\section{SAMPLES AND EXPERIMENTAL DETAILS}

A single phenol-derived activated carbon fiber (supplied by Kuraray Chemical Co.), with properties listed in Table $I$, is mounted on a mica substrate for conductivity measurements. The specific surface area of these fibers is denoted in the last two digits of the fiber name when multiplied by 100 in units of $\mathrm{m}^{2} / \mathrm{g}$ (see Table I). The mica substrate was set on a copper heat sink that was suspended onto a cryo-stage of an Air Products Cryogenics closed cycle $\mathrm{He}$ refrigerator (see Fig. 1). To measure the sample temperature, a thermometer was mounted on the opposite side of the copper heat sink. Electrical contact to the fibers was made with silver paint and four $0.5 \mathrm{~mm}$ diameter copper wires were thus attached for electrical contacts. A dc four-terminal method was used for the conductivity measurements with a constant $1 \mu \mathrm{A}$ current source. The effect of the temperature rise due to Joule heating was negligible because the measurements at room temperature showed the same resistivity over the current range $0.1-10 \mu \mathrm{A}$. The current direction was reversed during the measurements to check for possible thermoelectric effects in the contacts.

The inside of the system was pumped down to $10^{-3}$ Torr at room temperature to reduce the effects of gas adsorption, since in a preliminary measurement a remarkable decrease in photoconductivity was observed

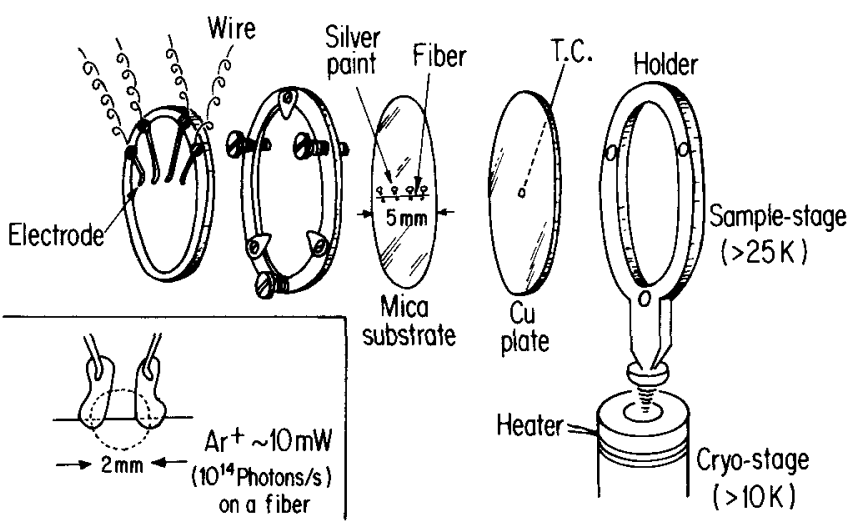

FIG. 1. Geometry of the ACF sample mounted on a mica substrate. The laser beam $\left(10 \mathrm{~mW} \mathrm{Ar}{ }^{+}\right.$ion laser $)$irradiates the indicated circular region (see inset), and $\simeq 10^{14}$ photons/s are incident on the fiber.

for ACFs exposed to air. In air, the room temperature photoconductivity diminished by a factor of 10 , compared to vacuum, although the intrinsic conductivity showed almost no change between exposure to air or vacuum. This adsorption is a physisorption process because the change in the photoconductivity was reversible. Effects of oxygen and water vapor have been investigated in terms of gas adsorption on carbon materials, ${ }^{2,3,8,9}$ where the intrinsic resistivity generally increased as a result of adsorption. In our experiments, water vapor is the most probable gas which changes the photoconductivity of the ACFs reversibly at room temperature because reversible physisorption usually occurs at temperatures below the boiling point of the adsorbates. Besides that, the effect of nitrogen is known to be negligible and oxygen is known to cause an irreversible chemisorption at room temperature. ${ }^{8} \mathrm{On}$ the other hand, a change in the intrinsic resistivity was observed when a fiber was baked in air at around $600 \mathrm{~K}$. The resistivity decreased by $10-15 \%$ as a result of the baking and the room temperature resistivity slowly returned to the initial value after exposure to air for one day. Thus the chemisorption of oxygen, which is not removed by pumping down, is indicated to take place in the ACFs. Our experimental results therefore

TABLE I. Properties of phenol-derived activated carbon fibers. ${ }^{33}$

\begin{tabular}{|c|c|c|c|c|}
\hline & FR10 & FR12 & FR15 & FR20 \\
\hline Specific surface area $\left(\mathrm{m}^{2} / \mathrm{g}\right)$ & 1000 & 1200 & 1500 & 2000 \\
\hline Pore radius $(\AA)$ & 9 & 10 & 12 & 16 \\
\hline Pore volume $\left(\mathrm{cm}^{3} / \mathrm{g}\right)$ & 0.22 & 0.35 & 0.50 & 0.75 \\
\hline Benzene adsorption capacity (wt.\%) & 22 & 35 & 45 & 65 \\
\hline Ash $(\mathrm{wt} . \%)$ & 0.03 & 0.03 & 0.03 & 0.04 \\
\hline Conductivity at room temperature $^{a}(\mathrm{~S} / \mathrm{cm})$ & $\simeq 10$ & $=20$ & $\simeq 25$ & $\simeq 35$ \\
\hline
\end{tabular}

${ }^{\mathrm{a}}$ From this paper. 
include some effects relevant to oxygen adsorption or surface oxidization.

Scanning electron microscopy (SEM) was used to determine the fiber diameters and to observe the macrostructure of the fibers. The SEM results of Fig. 2 show macroscopic pores with openings of about $500 \AA$ in the ACFs. Besides the macroscopic pores, the ACFs have microscopic pores with mean radii listed in Table I. As seen in Fig. 2, the fibers have a rather regular round cross section and a smooth surface.

The x-ray diffraction pattern for ACFs (Fig. 3) is broad, indicating an amorphous-like microstructure. An estimate for $L_{c}$ of about $10 \AA$ (about three interlayer spacings, the similar length as for Saran carbon ${ }^{1}$ ) was obtained from the linewidth of the $(002) x$-ray diffraction line for phenol-derived ACFs with a specific surface are of $1200 \mathrm{~m}^{2} / \mathrm{g}$.

We determined the dominant conduction type as $p$-type at room temperature by a rough measurement of the thermoelectric power, which was found to be small. The high temperature terminal in this measurement showed a negative potential (i.e., carrier type is positive) and the thermoelectric power was found to be about $1.7 \mu \mathrm{V} / \mathrm{K}$ at room temperature. Disordered carbon materials are known to be usually $p$-type. ${ }^{43,44}$

In the photoconductive measurements, an $\mathrm{Ar}$ ion laser beam (488 $\mathrm{nm}$ wavelength) with a $2 \mathrm{~mm}$ spot size and with $10 \mathrm{~mW}$ power was focused onto an entire ACF ( $\simeq 1 \mathrm{~mm}$ long, $\simeq 10 \mu \mathrm{m}$ diameter) through focusing optics and a quartz window (see Fig. 1). As shown in the figure, the beam covered the entire fiber (i.e., volume excitation), including a $1 \mathrm{~mm}$ interval between the two electrodes. The laser power was varied in the range $1-40 \mathrm{~mW}$ at each temperature $25,100,200$, and $290 \mathrm{~K}$ to determine the recombination kinetics. The number of incident photons on a fiber, for example in the

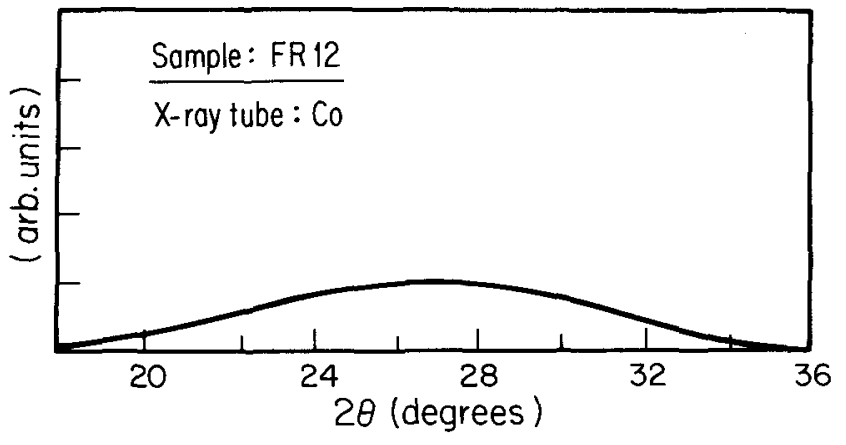

FIG. 3. X-ray diffraction pattern of ACF (FR12).

case of $10 \mathrm{~mW}$ incident laser power, was on the order of $10^{14} / \mathrm{s}$.

\section{RESULTS AND ANALYSIS}

\section{A. Intrinsic conductivity}

In this work, we use the term intrinsic conductivity to refer to the conductivity in the absence of light (dark conductivity), and the term intrinsic carriers is used to denote the carriers present in the absence of the light excitation. In the measurements of the temperaturedependence of the intrinsic conductivity $\sigma(T)$, a semiconductor-like positive temperature dependence is found (see Fig. 4). This kind of temperature dependence is also observed in other disordered carbons. $^{2,4,6,12,14,19,20,45,46}$ Table II shows room temperature conductivities of the ACFs and other disordered carbon materials. The magnitude of this conductivity and its temperature-dependence are similar to those of Saran carbon, ${ }^{1}$ active carbon rods, ${ }^{3}$ glassy carbons heat treated at about $1000-1100 \mathrm{~K},{ }^{12}$ and anthracene chars. ${ }^{45}$ The magnitude of the conductivity is higher than that of evaporated carbon films ${ }^{5}$ but lower than that of vapor-grown carbon fibers ${ }^{47}$ and PAN-based fibers. ${ }^{48}$
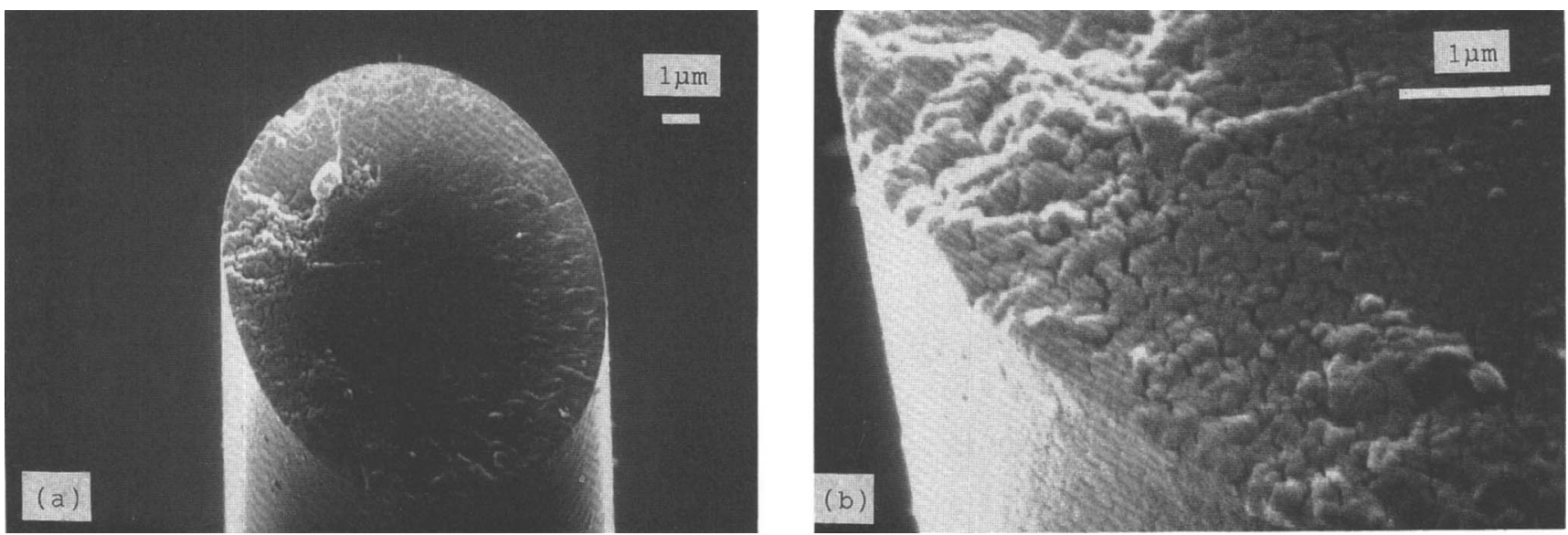

FIG. 2. Scanning electron microscopy photographs of a phenol-derived ACF: (a) cross section of a fiber, having a rather round shape and a smooth surface and (b) cross section with a higher magnification. The width of the crack-like openings is $\simeq 500 \AA$. The length scales in both photographs are given. 


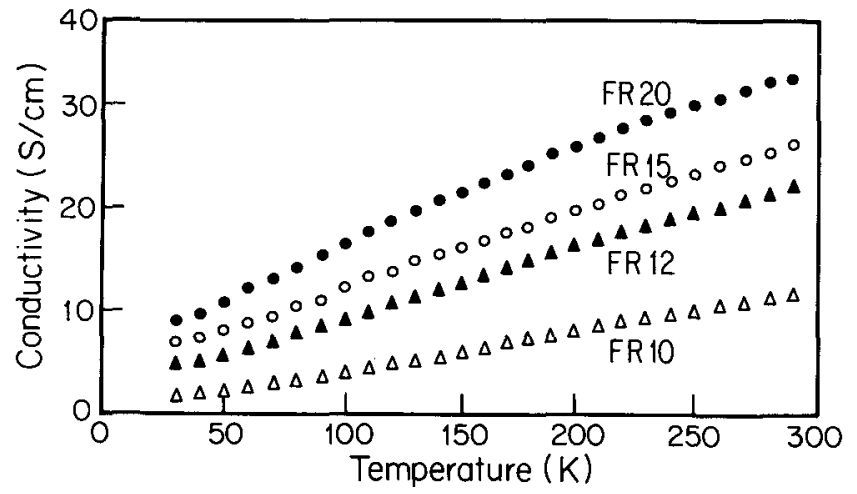

FIG. 4. Temperature-dependence of the "intrinsic" electrical conductivity for the four types of fibers.

One of the features of interest in Fig. 4 is the nearly linear temperature dependence of the conductivity for all ACFs in the studied temperature range. On the other hand, the slight departure from linearity gives information on the conduction mechanism discussed in Sec. IV. The dominant conduction mechanism in this material seems to be a thermal activation process in the studied temperature range.

However, at low temperatures (below $\simeq 40 \mathrm{~K}$ ), the conduction due to the thermal activation will be small and then hopping of carriers from one localized state to another (variable range hopping ${ }^{49}$ ) will be dominant. In this case, more disorder facilitates the hopping of carriers as the density of defect states near the Fermi level increases and the defects involved in the hopping process come closer to one another. Looking at the low temperature regime in Fig. 4, the fibers with more disorder (i.e., more specific surface area) have the higher conductivities. On the other hand, at high temperatures the thermal activation process will be dominant, as the carriers acquire sufficient energy to overcome the activation barrier.

According to this conduction mechanism, the conductivity is expected to be zero at $0 \mathrm{~K}$. However, in Fig. 4 this trend for the behavior of $\sigma(T)$ at lower temperatures is not clearly identified so that a lower tem- perature measurement is required to confirm the expected conductivity behavior in the low temperature limit. A fit to the $\sigma(T)$ is discussed further in Sec. IV.

\section{B. Photoconductivity}

In contrast with the intrinsic electrical conductivity, the photoconductivity exhibits a negative temperature dependence for all the ACFs that were investigated (see Fig. 5). This result is consistent with the behavior of the intrinsic conductivity in Fig. 4 because the recombination rate becomes larger as the intrinsic conductivity increases with temperature. Also of interest in Fig. 5 is the observation that the fibers with higher surface area also have higher photoconductivities. This fact suggests that the same basic conduction mechanism is operative for the photoconductivity as for the intrinsic conductivity. Namely, the photocarriers are also in localized states, and the fibers with more disorder have a smaller hopping range which leads to the higher conduction.

The decay curves of the photoconductivity show a nearly exponential fall with similar decay times, in agreement with previous work on pitch-based carbon fibers. ${ }^{42}$ All the fibers exhibit long decay times of about $0.3 \mathrm{~s}$, which are almost independent of temperature and not sensitive to the SSA of the fibers. In contrast to these long decay times, for usual semiconductors with free carriers, much faster decay times (on the order of $\mu \mathrm{sec}$ ) are observed.

The kinetics of the recombination process in the ACFs is different from that given in a previous report on an evaporated carbon film ${ }^{6}$ and carbon fibers ${ }^{42}$ where only the bimolecular recombination process was observed. The bimolecular recombination process is observed generally when the intrinsic carrier density is small and the photocarrier density becomes dominant. ${ }^{50-52}$ Figure 6 for the FR15 fiber shows the dependence of photoconductivity on the laser intensity on a $\log$-log scale for various temperatures (the other fibers exhibit the same trend). The significance of this figure is that it shows a change in the recombination kinetics

TABLE II. Conductivity values for various disordered carbons.

\begin{tabular}{lllr}
\hline \hline & $T_{H T}\left({ }^{\circ} \mathrm{C}\right)$ & Conductivity (S/cm) & Reference \\
\hline Saran carbon & $\simeq 720$ & $20-100$ & 1 \\
Active carbon rods & $\simeq 750$ & $\simeq 20$ & 3 \\
Glassy carbon & $\approx 750$ & $1-10$ & 12 \\
& $800-1000$ & $\simeq 0.1$ & 5 \\
Evaporated carbon film & & $\simeq 900$ & 47 \\
Vapor grown carbon fiber & 1400 & $\simeq 500$ & 53 \\
& As-grown & $15-150$ & $10-40$ \\
Phenol formaldehyde resin & $700-900$ & & This paper \\
Phenol ACFs & $800-1100$ & & \\
& & & \\
\hline \hline
\end{tabular}




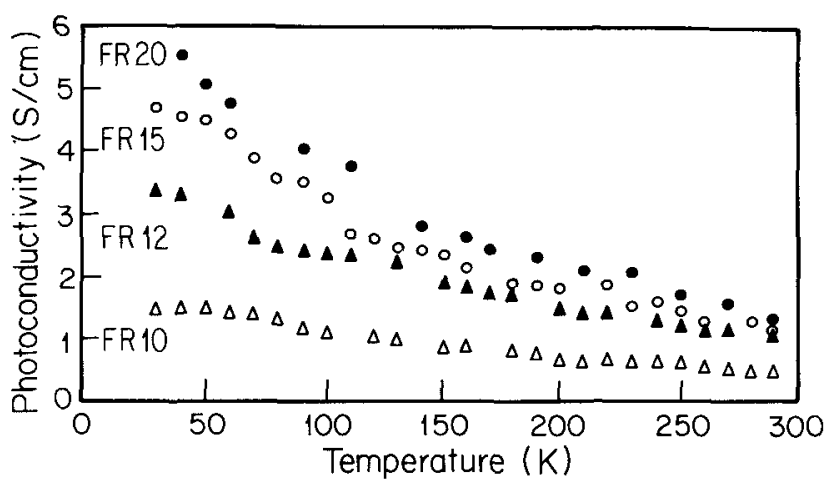

FIG. 5. Temperature-dependence of the photoconductivity for each of the fiber types.

according to the power law dependence for the photoconductivity, $\Delta \sigma \propto P^{\alpha}$ where $\Delta \sigma$ is the photoconductivity and $P$ is the incident laser power. If $\alpha=1$, the recombination process is monomolecular, while an exponent $\alpha=0.5$ indicates a bimolecular process. In general, a monomolecular process implies that the density of the intrinsic carriers is much higher than that of photocarriers so that the recombination is dominated by the intrinsic carriers. The inserted triangles in Fig. 6 indicate the slopes corresponding to the monomolecular and bimolecular processes. In the bimolecular process, recombination is limited by the availability of photogenerated holes $(\Delta p)$ to recombine with the photo-

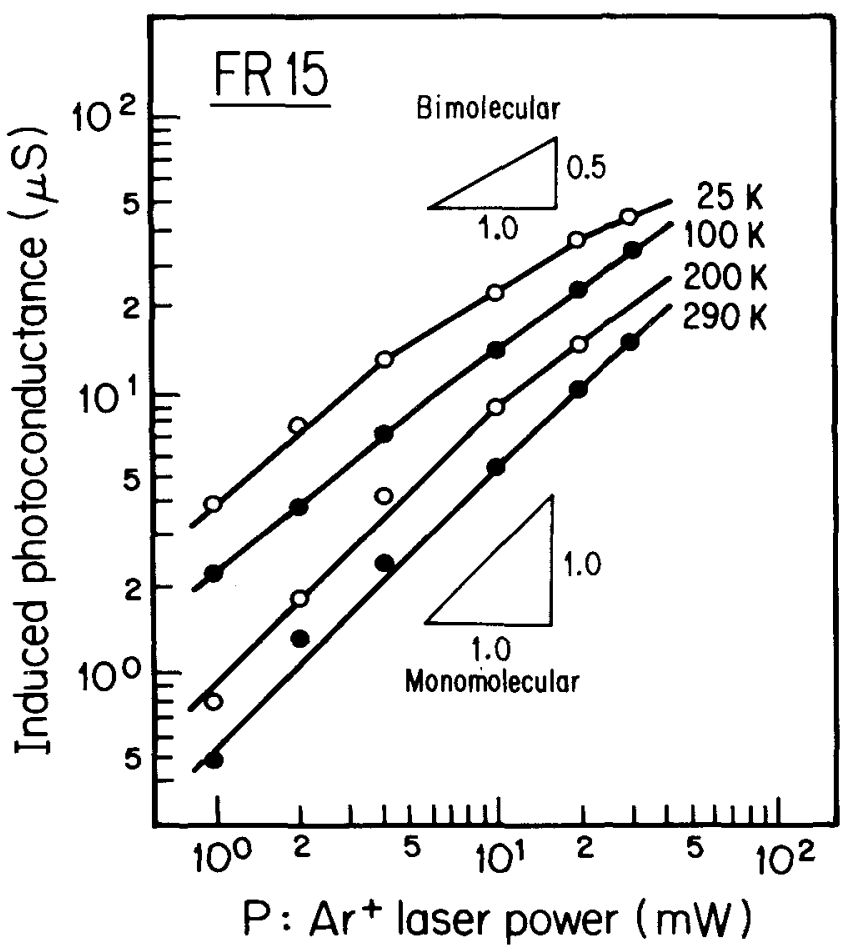

FIG. 6. Photoconductivity dependence on irradiation power at various temperatures for the FR15 fiber, plotted on a log-log scale. electrons $(\Delta n)$ so that recombination is proportional to the product $\Delta n \Delta p$ where $\Delta n=\Delta p$.

In contrast to the previous result for disordered carbon films, ${ }^{6}$ Fig. 6 clearly shows a monomolecular process $(\alpha=1.0)$ at high temperatures, while the onset of a bimolecular process $(\alpha=0.5)$ can be seen at low temperatures and at high laser powers. At $290 \mathrm{~K}$, the monomolecular process $(\alpha=1.0)$ is dominant at all laser powers shown. As the temperature is decreased, the slope decreases, indicating that the bimolecular process $(\alpha=0.5)$ becomes increasingly important and the monomolecular process $(\alpha=1.0)$ becomes less important. A typical case demonstrating the bimolecular process is the regime at $25 \mathrm{~K}$ with the laser power above $10 \mathrm{~mW}$, where the slope is close to 0.5 and the bimolecular process appears to be dominant. Slopes between 0.5 and 1.0 are observed for most of the data at $100 \mathrm{~K}$, suggesting that the contribution of the monomolecular process is comparable to that of the bimolecular process. This trend that the bimolecular process becomes dominant at low temperatures and at high laser powers is reasonable because the density of photocarriers becomes dominant relative to the intrinsic carrier density under those conditions.

Figure 7 shows more clearly the change in recombination kinetics. In this figure, the power (in $\Delta \sigma=$ $\left.A P^{\alpha}\right)$ is plotted versus the laser power $P$ at each temperature, where $A$ is a constant. In our experiments, the monomolecular process $(\alpha=1.0)$ was observed at high temperatures. This is consistent with the fact that the intrinsic conductivity is much larger than the photoconductivity at high temperatures. On the other hand, at low temperatures where the photoconductivity is comparable to the intrinsic conductivity, the power approaches 0.5 . This phenomenon suggests that the

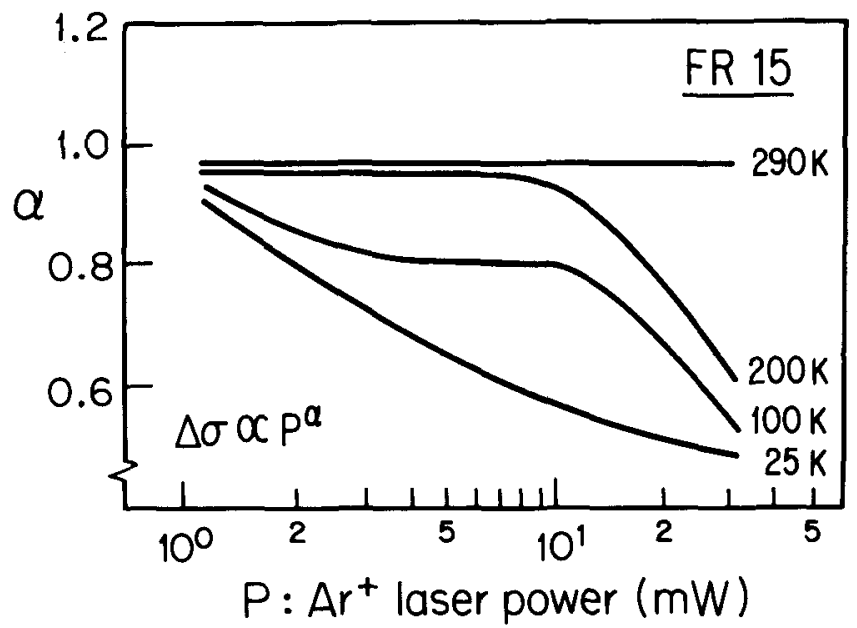

FIG. 7. Change in recombination kinetics at various incident power levels. $\alpha=1$ and 0.5 implies a monomolecular process or a bimolecular process, respectively. 
dominant carriers in the material are photocarriers at low temperatures, and as a result the main recombination process occurs between the photocarriers (i.e., between the photoelectrons and photoholes). In our experiments, both types of recombination kinetics were observed since the relative dominance of the intrinsic carrier concentration and of the photocarriers could be reversed, depending on the choice of incident laser power and on the temperature.

The magnitudes of the dependence on laser power of the photoconductivity $\Delta \sigma(T)$ relative to room temperature are shown in Fig. 8 (for the FR15 fiber) for three different temperatures. This figure indicates that the temperature dependence of the photoconductivity becomes smaller with increasing laser power. If the data are extrapolated to high laser power levels, the temperature-dependence would disappear at around $100 \mathrm{~mW}$. At $100 \mathrm{~mW}$ where the photoconductivity is essentially temperature-independent, the density of intrinsic carriers is negligible compared to the photocarriers induced at that power level $\left(\simeq 10^{15}\right.$ photons/s per fiber). In contrast, at low laser powers where the density of the induced photocarriers is small, the photoconductivity is rather temperature-dependent.

We note that the observed increase in conductivity during the laser pulse is not caused by a heating effect. If a heating effect caused the increase in conductivity, changes in the recombination kinetics would not be observed. Furthermore, a temperature rise caused by a heating effect would produce an almost temperature-

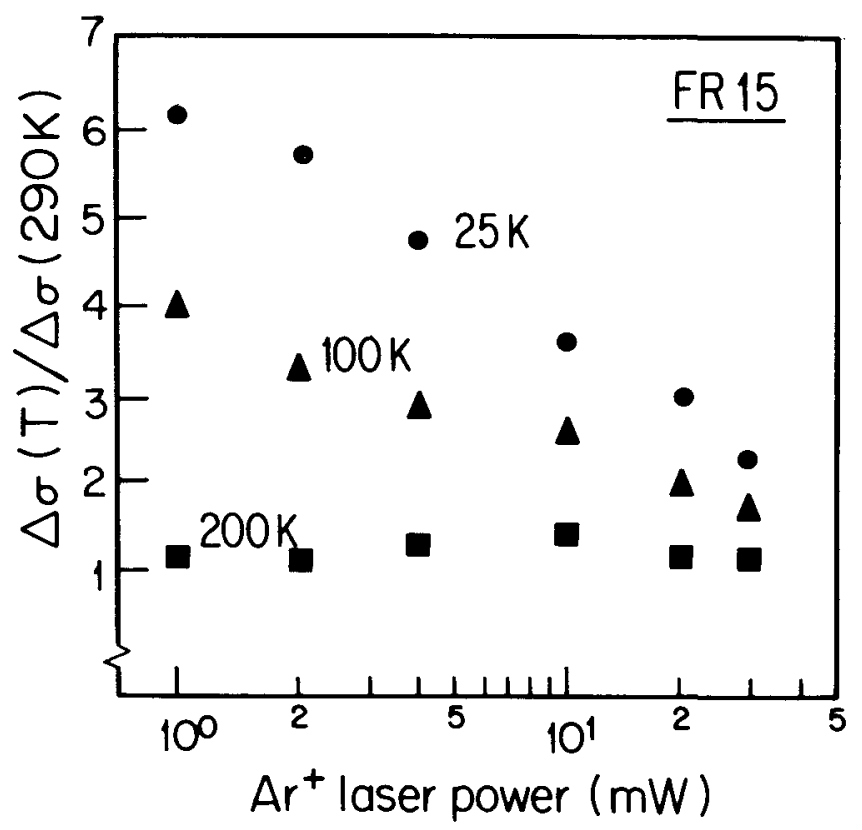

FIG. 8. Change in the temperature-dependence of the photoconductivity as a function of power level. An extrapolation to the high power limit indicates that the temperature-dependence disappears (the ratio becomes unity for all temperatures shown) at $\approx 100 \mathrm{~mW}$. independent increase in the conductivity. Because the $d \sigma / d T$ is nearly constant, as seen in Fig. 4, a constant conductivity deviation would be observed, provided that the temperature rise due to the incident laser is near constant over the studied temperatures. However in fact, the experiments show that the conductivities (Fig. 5) change by a factor of several over the temperature range $30-290 \mathrm{~K}$. For these reasons, we believe that the effect is not a heating effect but a photoeffect.

\section{DISCUSSION}

We have fit the temperature-dependent conductivity data to investigate the intrinsic conduction mechanisms. Figure 9 shows the fit of a model where two terms are assumed, a constant term and a temperaturedependent term $\sigma=\sigma_{\text {hop }}+\sigma_{\text {act }}$. The first term $\sigma_{\text {hop }}$ is associated with the variable range hopping process, but appears to be temperature-independent above $30 \mathrm{~K}$ for the ACFs studied in the present work. The temperature-dependent term $\sigma_{\text {act }}\left[=\sigma_{\text {act }}^{\circ} \exp (-E / k T)\right]$ is written as a simple activation term. The fits for the various fibers to this functional form are shown in Fig. 9 and values for $\sigma_{\text {hop }}$ and $\sigma_{\text {act }}$ are given in Table III. The term due to the hopping conduction $\sigma_{\text {hop }}$ is found to increase strongly with increasing specific surface area (SSA), though we have not been able to find the functional dependence of $\sigma_{\text {hop }}$ on SSA. The temperaturedependence of the intrinsic conductivity then comes from the temperature-dependent term $\left(\sigma_{\text {act }}\right)$ based on a thermal activation process. From the fits in Fig. 9, we see that the activation energy $E$ decreases with increasing SSA, but because of the exponential dependence on $E$, the effect of increasing the SSA is quite large. The net effect is that the temperature-dependent term in-

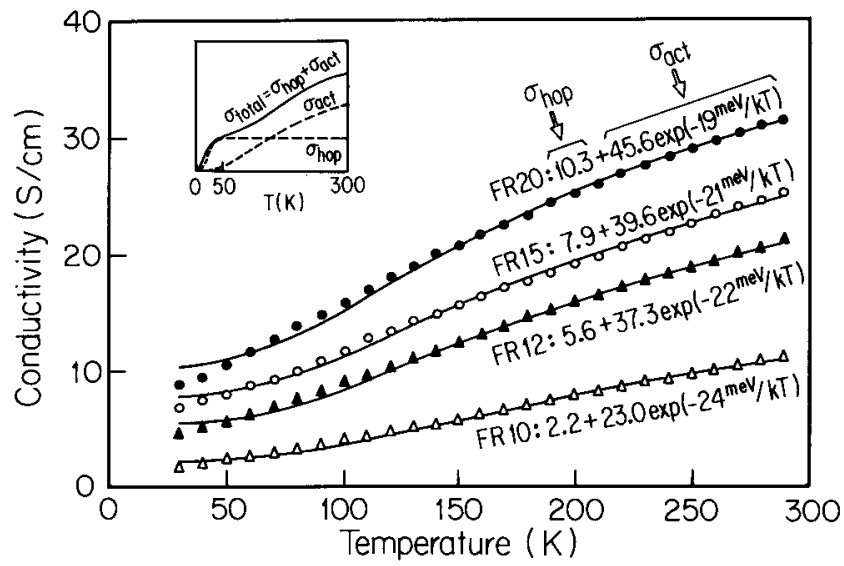

FIG. 9. Calculation of the "intrinsic" conductivities based on a conduction model. The inset indicates the conduction model schematically. The model consists of two terms based on a thermal activation process $\left(\sigma_{\text {act }}\right)$ and a hopping process $\left(\sigma_{\text {hop }}\right)$. The hopping process is almost constant over the studied temperature range. 
TABLE III. Fitting ${ }^{\text {a }}$ values for the conductivity.

\begin{tabular}{lccc}
\hline \hline & & \multicolumn{2}{c}{$\sigma_{\text {act }}(\mathrm{S} / \mathrm{cm})$} \\
\cline { 3 - 4 } & $\sigma_{\text {hop }}(\mathrm{S} / \mathrm{cm})$ & $\sigma_{\text {act }}^{\circ}(\mathrm{S} / \mathrm{cm})$ & $E(\mathrm{meV})$ \\
\hline FR10 & 2.2 & 23.0 & 24 \\
FR12 & 5.6 & 37.3 & 22 \\
FR15 & 7.9 & 39.6 & 21 \\
FR20 & 10.3 & 45.6 & 19 \\
\hline \hline
\end{tabular}

${ }^{\mathrm{a}} \sigma(T)=\sigma_{\mathrm{hop}}+\sigma_{\mathrm{act}}=\sigma_{\mathrm{hop}}+\sigma_{\mathrm{act}}^{\mathrm{o}} \exp (-E / k T)$.

creases more rapidly with SSA than $\sigma_{\text {hop }}$. For example, the magnitude of $\sigma_{\text {hop }}$ at room temperature is only 15$25 \%$ of $\sigma_{\text {act }}$ for all the fibers. At high temperatures, satisfactory agreement between the data and the model is shown in Fig. 9, although the fit is not good at low temperatures. In terms of the prefactor $\sigma_{\text {act }}^{\circ}$ of the thermal activation term $\sigma_{\text {act }}$, an increase (from 23.0 to $45.6 \mathrm{~S} / \mathrm{cm}$ ) with SSA (from 1000 to $2000 \mathrm{~m}^{2} / \mathrm{g}$ ) is observed. This trend is reasonable since the density of states near the Fermi level increases with increasing SSA. If the conductivity is described by two terms, there will be two main conduction mechanisms in our material.

With regard to the mechanism for the increase in the photoconductivity at low temperatures (Fig. 5), an increase in the photocarrier concentration is required if the photocarriers are also in localized states and as a result their mobility decreases with decreasing temperature. There can be two explanations for the increase in the photocarrier density. One explanation is that a decrease in the mobility of the photocarriers at low temperatures leads to a lower recombination rate. If carriers become less mobile, the probability for carriers to meet each other and to recombine is reduced.

The other explanation is that thermally excited intrinsic carriers play a main role in the recombination process. In this model, specific mobile carriers that are excited to extended states dominate the recombination process. Because the concentration of these excited carriers is temperature-dependent, the decrease in the concentration of these carriers at low temperatures leads to a lower recombination rate, resulting in a high density of the photocarriers. It is difficult to specify here which mechanism is dominant. It depends on whether the states of the carriers in this disordered carbon are all localized or whether they have some extended states.

In both cases, the density of photocarriers is expected to become large at low temperatures. The photoconductivity, however, is expected to show a decrease as the temperature is further lowered because the carriers become less mobile and because the density of photocarriers eventually should reach a finite value, depending on the laser power. In fact, the FR10 fiber seems to show a saturation in the photoconductivity at $\simeq 50 \mathrm{~K}$ in Fig. 5. This idea predicts that the photoconductivity and the intrinsic conductivity will eventually become zero at $0 \mathrm{~K}$.

Assuming an appropriate value of the mobility, it is possible to estimate the densities of the intrinsic carriers and of the photocarriers. For the mobility in the localized states, $\simeq 1 \mathrm{~cm}^{2} / \mathrm{Vs}$ can be taken based on the calculated mobility of a phenol resin ${ }^{53}$ for which the conductivity and the heat-treatment temperature were about the same as for the ACFs. Then the density of the intrinsic carriers and of the photocarriers at room temperature are estimated to be $\simeq 10^{21} / \mathrm{cm}^{3}$ and $\simeq 10^{20} / \mathrm{cm}^{3}$ (in the case of $10 \mathrm{~mW}$ laser power), respectively, assuming that the majority of carriers exist in trapped states and that the intrinsic carriers and the photocarriers have a similar mobility. This magnitude of the intrinsic carrier density is comparable to the value of $\simeq 10^{20} / \mathrm{cm}^{3}$ obtained by the Hall and ESR ${ }^{53}$ measurements of a phenol resin. These values are also reasonable because Fig. 8 indicates that about $100 \mathrm{~mW}$ of incident light would generate more photocarriers than the intrinsic carriers.

\section{CONCLUSIONS}

Measurements of the intrinsic conductivity and the photoconductivity of activated carbon fibers have been carried out over the temperature range $30-290 \mathrm{~K}$. The temperature-dependence is positive for the intrinsic conductivity and negative for the photoconductivity. The decay time of the photoconductivity is about $0.3 \mathrm{~s}$. The recombination kinetics change from a monomolecular process to a bimolecular process with decreasing temperature and increasing photon intensity. It is assumed that most intrinsic carriers and photocarriers exist in a high density of localized states in this material. The main conduction mechanism is assumed to be variable range hopping at low temperatures. At high temperatures either short-range hopping or thermal activation of carriers over the mobility edge can be operative. An activation energy of about $20 \mathrm{meV}$ for the temperature-dependent term in the intrinsic conductivity fits the experimental data well at high temperatures. The densities of the intrinsic carriers and the photocarriers at room temperature are estimated to be $\simeq 10^{21} / \mathrm{cm}^{3}$ and $\simeq 10^{20} / \mathrm{cm}^{3}$ (in the case of $10 \mathrm{~mW}$ laser power), respectively. Both the intrinsic conductivity and the photoconductivity are expected to approach zero as temperature approaches $0 \mathrm{~K}$. We have found that photoconductivity measurements, in combination with conductivity measurements, provide valuable information for understanding the electronic properties of disordered carbon materials. 


\section{ACKNOWLEDGMENTS}

We would like to thank Dr. G. Dresselhaus, Dr. I. Ohana, and Professor M. Endo for enlightening conversations, Dr. A. M. Rao for help with the experiments, and A.W. Fung and S. L. di Vittorio for both. We gratefully acknowledge support from Sumitomo Metal Industries (K.K.) and Lawrence Livermore Laboratory Subcontract \#B130530 (M.S. D.).

\section{REFERENCES}

${ }^{1}$ J. R. Dacey, D. F. Quinn, and J.T. Gallagher, Carbon 4, 73 (1966).

${ }^{2}$ R. McIntosh, R. S. Haines, and G. C. Benson, J. Chem. Phys. 15, 17 (1947).

${ }^{3}$ W.W. Smeltzer and R. McIntosh, Can. J. Chem. 31, 1239 (1953).

${ }^{4}$ M. D. Blue and G. G. Danielson, J. Appl. Phys. 28, 583 (1957).

${ }^{5}$ T. Hanawa and J. Kakinoki, Carbon 1, 403 (1964).

${ }^{6}$ J. M. MacFarlane, I. S. Mclintock, and J. C. Orr, Phys. Status Solidi (a) 3, K239 (1970).

${ }^{7}$ S. Mizushima and Y. Hirabayashi, Carbon 6, 123 (1968).

${ }^{8}$ I. S. Mclintock and J. C. Orr, Carbon 6, 309 (1968).

${ }^{9}$ V.R. Deitz and E. F. MacFarlane, in Proceedings of the Fifth Carbon Conference (Pergamon Press, Oxford, 1963), Vol. 2, p. 219.

${ }^{10}$ I. S. Mclintock and J. C. Orr, Carbon 5, 291 (1967).

${ }^{11} \mathrm{H}$. Hirabayashi and H. Toyoda, in Proceedings of the Fourth Carbon Conference (Pergamon Press, Oxford, 1961), p. 227.

${ }^{12}$ F. Carmona, P. Delhaes, G. Keryer, and J.P. Manceau, Solid State Commun. 14, 1183 (1974).

${ }^{13}$ R. R. Saxena and R. H. Bragg, J. Non-Cryst. Solids 28, 45 (1978).

${ }^{14}$ D. F. Baker and R. H. Bragg, Phys. Rev. B 28, 2219 (1983).

${ }^{15}$ J.W. Armstrong, C. Lackson, and H. Marsh, Carbon 2, 239 (1964).

${ }^{16}$ B. D. McMichael, E. A. Kmetko, and S. Mrozowski, J. Opt. Soc. Am. 44, 26 (1954).

${ }^{17}$ P. L. Walker, L. G. Austin, and J. J. Tietjen, Carbon 2, 1710 (1965).

${ }^{18}$ Y. Maruyama and H. Inokuchi, Chem. Soc. of Jpn. Bull. 39, 1418 (1966).

${ }^{19}$ E. A. Davis and R. F. Shaw, J. Non-Cryst. Solids 2, 406 (1970).

${ }^{20}$ W. Bücker, J. Non-Cryst. Solids 12, 115 (1973).

${ }^{21}$ S. Mrozowski, Phys. Rev. 77, 838 (1950).

${ }^{22}$ S. Mrozowski, Carbon 3, 305 (1965).

${ }^{23}$ S. Mrozowski, Carbon 6, 841 (1968).
${ }^{24}$ S. Mrozowski, Phys. Rev. 85, 609 (1952).

${ }^{25}$ S. Mrozowski, Carbon 9, 97 (1971).

${ }^{26}$ S. Mrozowski, Carbon 11, 433 (1973).

${ }^{27}$ S. Mrozowski, J. Low Temp. Phys. 35, 231 (1979).

${ }^{28}$ H. Nagata and T. Yoshida, Chemical Economics, 44 (April 1977).

${ }^{29}$ S. Ikegami and K. Shimazaki, Fuel and Combustion 54, 2 (1987).

${ }^{30} \mathrm{~N}$. Ishizaki, Chemical Engineering, 24 (July 1984).

${ }^{31}$ K. Tai and N. Shishido, Polymer Processing 35, 384 (1986).

${ }^{32}$ G. N. Arons and R. N. Macnair, Textile Res. J. 42, 60 (1972).

${ }^{33}$ E. Tanaka, Fuel and Combustion 54, 241 (1987).

${ }^{34}$ A. Yoshida, I. Tanahashi, Y. Takeuchi, and A. Nishino, IEEE CHMT-10, 100 (1987).

${ }^{35}$ N. Kasaoka, Y. Sakata, E. Tanaka, and R. Naito, J. Chem. Soc. Jpn. 5, 990 (1987).

${ }^{36}$ G. M. Nieh and D.T. Grow, J. Colloid Interface Sci. 119, 280 (1987).

${ }^{37}$ J.W. Herrick, in 23rd Ann. Tech. Conf. SPI Reinforced Plastics/Composites, 16A (1968).

${ }^{38}$ Y. Komatsubara, S. Ida, H. Fujitsu, and I. Mochida, Fuel 63, 1738 (1984).

${ }^{39}$ I. Mochida, M. Ogaki, H. Fujitsu, Y. Komatsubara, and S. Ida, Fuel 64, 1054 (1985).

${ }^{40}$ G. G. Jayson, T. A. Lawless, and D. J. Fairhurst, J. Colloid Interface Sci. 86, 379 (1982).

${ }^{41}$ K. Kaneko and N. Shindo, Carbon 27, 815 (1989).

${ }^{42}$ J. Steinbeck, G. Braunstein, M. S. Dresselhaus, G. Dresselhaus, and T. Venkatesan, in Extended Abstracts No. 8, Graphite Intercalation Compounds, edited by M.S. Dresselhaus, G. Dresselhaus, and S. A. Solin (Materials Research Society, Pittsburgh, PA, 1986), p. 129.

${ }^{43}$ S. Mrozowski and A. Chaberski, Phys. Rev. 104, 74 (1956).

${ }^{44}$ C. A. Klein, J. Appl. Phys. 35, 2947 (1964).

${ }^{45} \mathrm{P}$. Delhaes and F. Carmona, in Chemistry and Physics of Carbon (Marcel Dekker, New York, 1981), Vol. 17.

${ }^{46}$ S. L. di Vittorio, M. S. Dresselhaus, M. Endo, J-P. Issi, L. Piraux, and V. Bayot, J. Mater. Res. 6, 778 (1991).

${ }^{47}$ J. Heremans, Carbon 23, 431 (1985).

${ }^{48}$ I. L. Spain, K. J. Volin, H. A. Goldberg, and I. Kalnin, J. Phys. Chem. Solids 44, 839 (1983).

${ }^{49}$ N. Mott, Conduction in Non-Crystalline Materials (Oxford University Press, 1987).

${ }^{50}$ R. H. Bube, Photoconductivity of Solids (Wiley, New York, 1960).

${ }^{51} \mathrm{~A}$. Rose, Concepts in Photoconductivity and Allied Problems (Interscience Publishers, New York, 1963).

${ }^{52}$ Photoconductivity and Related Phenomena, edited by J. Mort and D. M. Pai (Elsevier, 1976).

${ }^{53}$ W. Bücker, J. Non-Cryst. Solids 18, 11 (1975). 